\title{
GEM based TPC for the high intensity beam at J-PARC
}

\author{
Shin Hyung Kim ${ }^{1 *}$, Jung Keun Ahn ${ }^{1}$, Hiroyuki Sako², Yudai Ichikawa ${ }^{2}$, Hitoshi \\ Sugimura ${ }^{2}$ \\ ${ }^{1}$ Department of Physics, Korea University, Seoul 02841, Korea \\ ${ }^{2}$ Advanced Science Research Center, Japan Atomic Energy Agency, Tokai 319-1184, Japan \\ E-mail: shkimenuclear.korea.ac.kr
}

\begin{abstract}
The time projection chamber(HypTPC) has been developed for the H-dibaryon search experiment via $\left(K^{-}, K^{+}\right)$reactions at J-PARC. The high rate secondary beam up to $10^{6} \mathrm{~Hz}$ from the world highest intensity proton beam should be taken into account. The HypTPC has the octagonal drift volume defined by the field cage, the cathode plane at the top and the amplification region at the bottom. The drift length is $55 \mathrm{~cm}$. The Helmholtz type superconducting magnet applies the uniform magnetic field to the drift volume of the TPC. The target is located at $143 \mathrm{~mm}$ upstream from the center of TPC inside the drift volume. The outermost gas vessel is filled with P-10 gas. To avoid the charge build-up of field cage in the high rate beam, we cut the exposed insulator part of field cage around beam through region. When charged particles pass through the gas volume, ionized electrons along the track drift downward to first meet the gating grid plane and then triple GEMs, which are adopted to reduce ion back flows in the high rate beam. The amplified electron signals from GEM can be read out by almost 6000 pads which has a concentric configuration around the target. The GET(Generic Electronic System for TPCs) is used for the data acquisition. We adopted the partial readout mode and the zero suppression to reduce the dead time of DAQ combined with $\left(K^{-}, K^{+}\right)$trigger at the high beam intensity. We will report commissioning of the HypTPC and the DAQ performance test.
\end{abstract}

XVII International Conference on Hadron Spectroscopy and Structure - Hadron2017

25-29 September, 2017

University of Salamanca, Salamanca, Spain

${ }^{*}$ Speaker. 


\section{Introduction}

The J-PARC E42 experiment was proposed to search for the H-dibaryon via $\left(K^{-}, K^{+}\right)$reactions with the world highest intense $K^{-}$beam and a high resolution Hyperon spectrometer mainly consisting of a time projection chamber(HypTPC) and a superconducting magnet[1]. The HypTPC was newly developed to detect the decay products of $\mathrm{H}$-dibaryon such as $\Lambda \Lambda, \Lambda p \pi^{-}$, or $\Xi^{-} p$. The high rate secondary beam up to $10^{6} \mathrm{~Hz}$ was taken into account in the TPC design. In this paper, we report the commisioning of the HypTPC and the data acquisition test results.

\section{HypTPC Structure}

The HypTPC has the octagonal prism shaped gas vessel which contains $\mathrm{P}-10(\mathrm{Ar} 90 \%+$ $\mathrm{CH}_{4} 10 \%$ ) gas as shown in Fig. 1(a). The Fig. 1(b) shows the drift volume confined by the cathode plane, the field cage structure, and the amplification region. The target holder structure is located at $143 \mathrm{~mm}$ upstream from the center inside the drift volume, where the diamond target settles down. The Fig. 1(c) presents the amplification region components; the gating grid plane, the triple GEM layer, the pad plane, and the conversion board in an order from top to bottom.

When charged particles go through the gas volume, the ionized electrons drift downwards along the applied electric field. The electrons are amplified by passing the triple GEM with a gain of an order of $10^{4}$ and collected by 5768 pads which are arranged concentrically around the target position. The conversion board at the bottom of the chamber guides the electron signals to the electronics for the data acquisition.
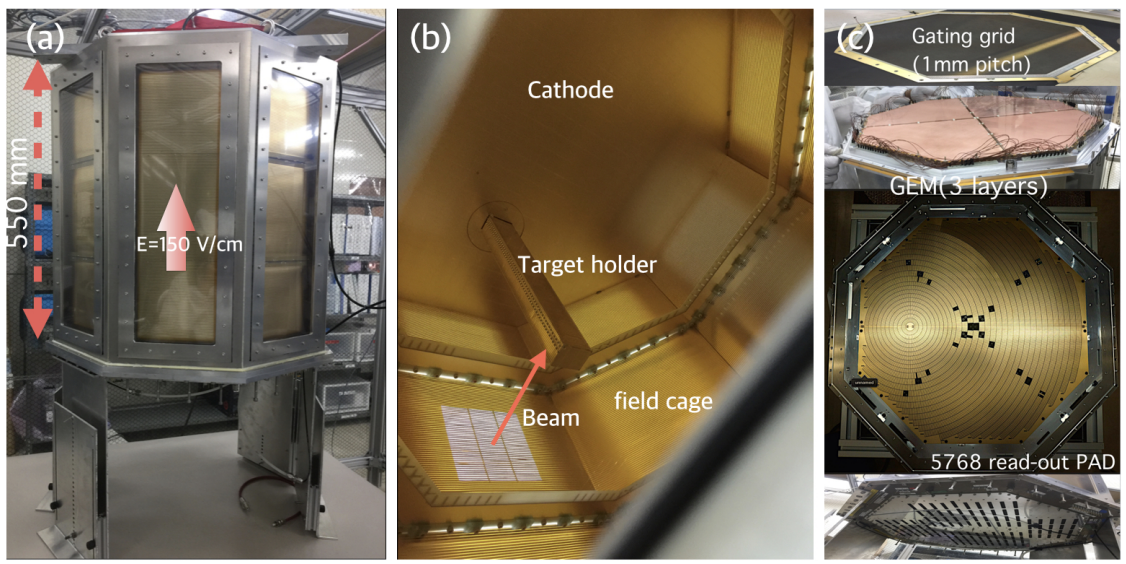

Figure 1: The structure of HypTPC. The detailed description is in the text.

In order to avoid the charge build-up in the high rate beam environment, which causes the field distortion, we cut the exposed insulation part of the field cage in the beam-through region as shown in Fig. 1(b). The ion backflow effect also has an impact on the field uniformity especially in the high rate beam condition. The gating grid system with the GEM was adopated to suppress the ion backflow rate[2]. The gating grid wire is made of $A u$-plated $\mathrm{Cu}$-Be wires of $50 \mu \mathrm{m}$ in diameter and the pitch is $1 \mathrm{~mm}$. When the gate is closed, alternate wires are biased to $V_{C O M} \pm V_{\text {GATE }}$, where 
$V_{C O M}$ is the common voltage and $V_{G A T E}$ is the alternate biased voltage. When the trigger comes in, the gate is opened with $V_{G A T E}=0$ as depicted in Fig. 3(a).

\section{Comissioning of HypTPC}

The basic performance of the HypTPC has been tested using a $460 \mathrm{MeV}$ position beam at ELPH, Tohoku University. We could successfully reconstruct the position track as shown in Fig. 2. The position resolution is around $500 \mu \mathrm{m}$, which corresponds to $300 \mu \mathrm{m}$ under the magnetic field of $1 \mathrm{~T}$ [2], and the drift velocity is $4.5 \mathrm{~cm} / \mu \mathrm{s}$ at the drift field of $130 \mathrm{~V} / \mathrm{cm}$. The gating grid operation has been also tested and the ion backflow is drastically suppressed from $V_{\text {GATE }}=30 \mathrm{~V}$ as shown in Fig. 3(b) and 4(a).
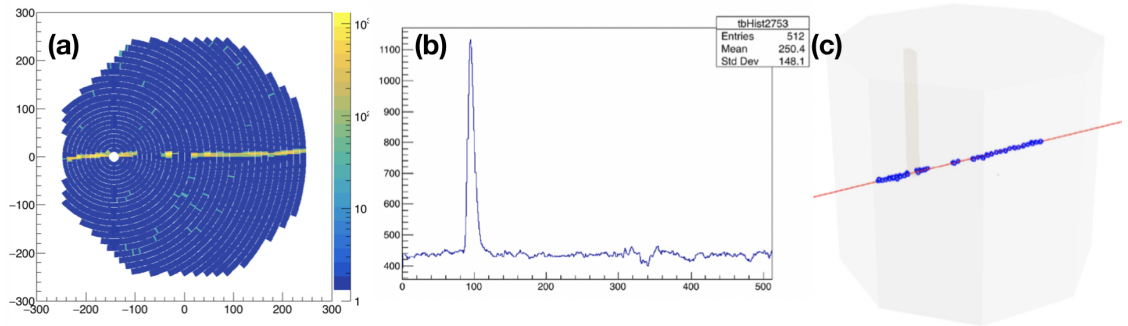

Figure 2: The positron track on the pad plane(a) and the typical pad signal(b). By combining the (a) and (b), the three dimensional track is reconstruced(c).

(a)

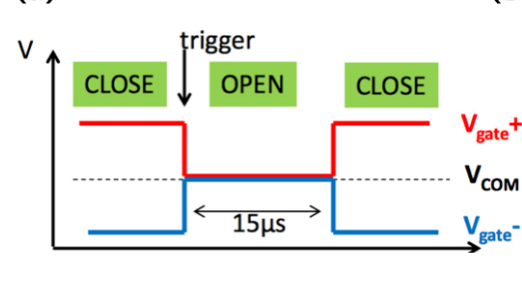

(b)

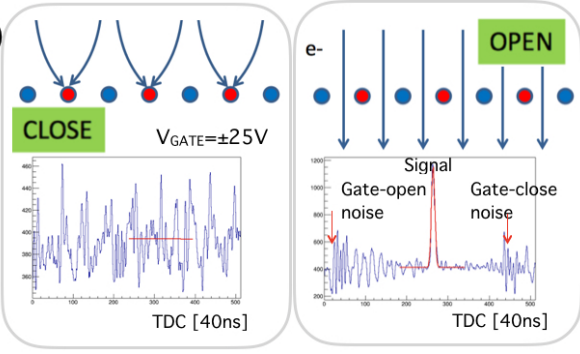

Figure 3: The gating grid operation is described(a) and its performance is tested using the beam(b).

We measured the ion backflow rate and the gain, respectively, with respect to the voltage applied to each GEM $\left(V_{G E M}\right)$ using the $\beta$ ray source. As the GEM voltage increases, the ion backflow rate decreases and the gain goes up as presented in Fig. 4(b), (c). The GEM discharge rate also increases with higher GEM voltage, so we need an optimization for the GEM voltage.

\section{Data Acquisition System}

The GET(Generic Electronic System for TPCs)[3] is adopted for the HypTPC data acquisition(DAQ). The signals from the HypTPC pads are transfered to the AsAd(ASIC and ADC) board via the conversion board and the protection board(ZAP), and are amplified, shaped, sampled, and 

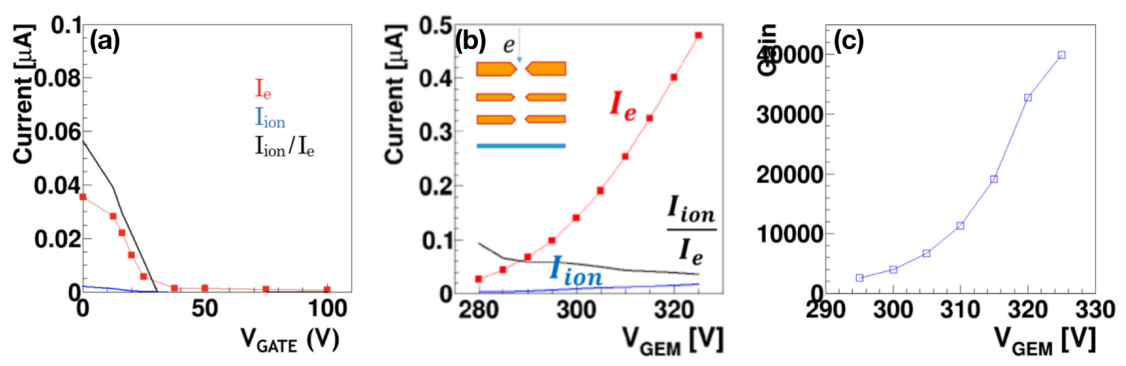

Figure 4: The ion backflow rate is measured with respect to $V_{G A T E}$ using the positron beam(a). The ion backflow rate(b) and the gain(b) are evalulated with respect to $V_{G E M}$ using the $\beta$ ray source.

digitized in AsAd board. The $\mathrm{CoBo}$ (Concentration Board) processes the digitized signal and saves the data. The partial readout mode selects the signal in the AsAd board to send to CoBo and the zero suppression further reduces the digitized data in the CoBo to save on DAQ PC. We can deal with the high trigger rate following by the high intensity beam up to several $\mathrm{kHz}$ by applying the partial readout mode and the zero suppression at the same time(Fig. 5).
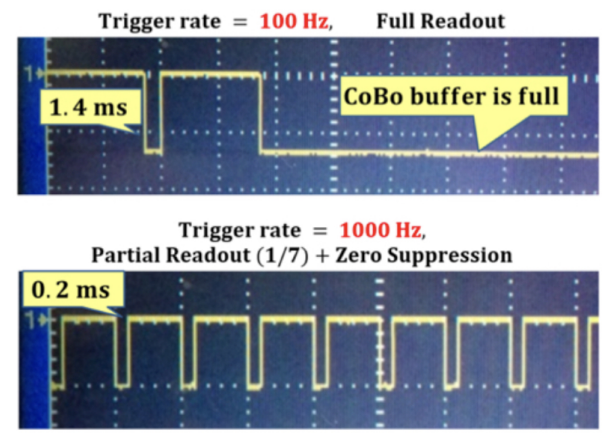

Figure 5: The busy time of the HypTPC DAQ system is shown in the full readout mode(top) and in the partial readout mode and the zero supperssion(bottom).

\section{Summary}

The time projection chamber(HypTPC), a central tracking device in the H-dibaryon search experiment at J-PARC, has been newly developed with a special care of the high intensity beam. The basic performance of HypTPC has been tested with the positron beam and the $\beta$ ray source and the DAQ system has been optimized to the high intensity beam.

\section{References}

[1] J.K. Ahn and K. Imai, J-PARC Proposal E42, Search for the H-dibaryon with a Large Acceptance Hyperon Spectrometer.

[2] H. Sako et al., Nucl. Instrum. Methods A 763 (2014) 65-81.

[3] J. Giovinazzo et al., Nucl. Instrum. Methods A 840 (2016) 15-27. 